\title{
Ultrasound Guided Aspiration of Lateral Parameniscal 㗊德 Cysts Causing Iliotibial Band Friction Syndrome
}

\author{
Fajin Dong MD ${ }^{1}$, Alfonso Matthew BS ${ }^{2}$, Lei Zhang ${ }^{1}$, Carlos M Barrera BS ${ }^{3}$, Jinfeng Xu MD ${ }^{1}$, Evan R Finkelstein MD ${ }^{4}$, \\ Harry G Greditzer $\mathrm{MD}^{5}$ and Jean Jose $\mathrm{DO}^{* 4}$ \\ ${ }^{1}$ Department of Ultrasound, First Affiliated Hospital of Southern University of Science and Technology, Second Clinical College of Jinan University, China. \\ ${ }^{2}$ Herbert Wertheim College of Medicine - Florida International University, USA
}

${ }^{3}$ Miller School of Medicine, University of Miami, USA

${ }^{4}$ Department of Radiology, University of Miami Miller School of Medicine, USA

${ }^{5}$ Department of Radiology \& Imaging, The Hospital for Special Surgery, USA

Received: 㘹: September 29, 2018; Published: 眥: October 04, 2018

*Corresponding author: Jean Jose DO, Department of Radiology, University of Miami Miller School of Medicine, Jackson Memorial Hospital, Miami, FL, USA

Jinfeng Xu, M.D, Department of Ultrasound, First Affiliated Hospital of Southern University of Science and Technology, Second Clinical College of Jinan University, Shenzhen Medical Ultrasound Engineering Center Shenzhen People's Hospital, Shenzhen, 518020, PR, China

\begin{abstract}
The purpose of this article is to provide an overview of parameniscal cyst pathology, diagnosis, and treatment. The article will discuss both established treatment modalities and a novel technique for treatment of parameniscal cysts in patients with concomittant Iliotibial Band Friction Syndrome. In this method, operators scan using a high-frequency linear ultrasound transducer with the scan plane corresponding to the anatomic coronal plane. They then place a 25-gauge needle along the anterolateral margin of the lateral meniscus for aspiration of the parameniscal cyst. Next, clinicians inject a standardized therapeutic mixture of anesthetic and long-acting corticosteroid into the undersurface of the adjacent iliotibial band. Distention of the bursa is the determining factor for a successful injection. Ultrasound allows confirmation of correct injection placement, resulting in increased accuracy and improved patient.
\end{abstract}

Keywords: Ultrasound Guided Aspiration; Ultrasound Guided Injection; Knee Ultrasound; Lateral Parameniscal Cyst; Iliotibial Band Friction Syndrome

\section{Introduction}

\section{Pathology}

Meniscal cysts are an accumulation of synovial fluid within a sac present in the knee joint. The cysts may be separated into two types, parameniscal and intrameniscal. Both types may be present simultaneously [1]. Meniscal cysts represent about $1 \%$ to $10 \%$ of all meniscal pathology. They are caused by meniscal tears resulting in one-way valves that trap synovial fluid and are most commonly associated with horizontal cleavage tears of the lateral meniscus. Thought to be the result of age-related meniscal degeneration, meniscal cysts have now been diagnosed in adolescents and pediatric patients [2]. Intrameniscal cysts are found within the body or substance of the meniscus (Figure 1). Parameniscal cysts are classified as lateral or medial depending on from which meniscus the cyst protrudes. Meniscal cysts may be considered secondary indirect signs of meniscal tears [3]. Even when meniscal tears are not visualized, parameniscal cysts have a highly specific association with meniscal tears. Rutten et al. found coexisting meniscal tears in $85 \%$ of patients with parameniscal cysts [4]. Bergin et al. found parameniscal cysts in $7 \%$ of patients with meniscal tears [5].

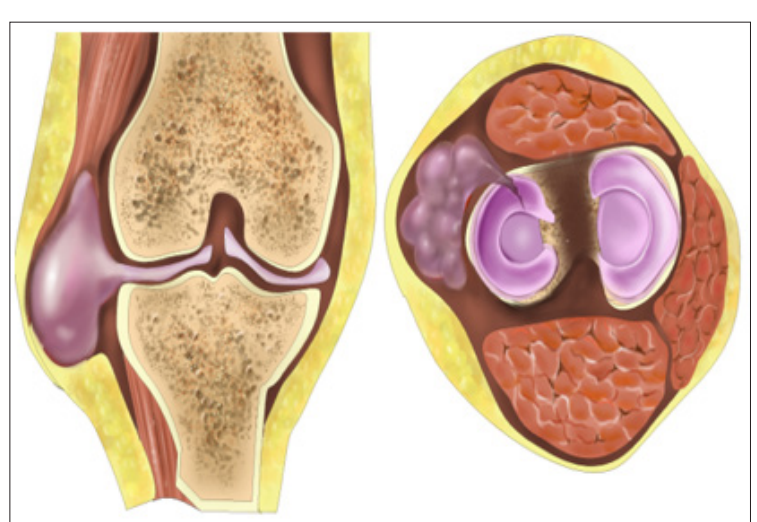

Figure 1: Intrameniscal cysts are found within the body or substance of the meniscus.

Iliotibial band friction syndrome (ITBS) involves the iliotibial band. The iliotibial band is a thick band of fascia lata that runs along the lateral thigh, originates at the iliac crest and inserts into the anterolateral aspect of the tibia [6]. ITBS is a multifactorial over- 
use injury. It is believe to be caused, in part, by friction between the distal part of the band and the lateral femoral condyle during repeated flexion and extension, sometimes resulting in formation of a pathological bursa $[7,8]$. This results in inflammation $[6,9,10]$. Symptoms are most acute when the knee is at 30-degree flexion, representing the point of maximal frictional force between the ITB and the condyle [9]. The main symptom is pain or tenderness on the lateral side of the knee that may extend along the length of the iliotibial band on the lateral side of the thigh. There are 3 layers of the lateral knee [10]. The first layer is the most superficial and contains the iliotibial band anteriorly and the biceps tendon posteriorly with associated fascial expansions in the thigh and calf. The second layer contains the lateral patellar retinaculum and the patellofemoral ligaments anteriorly and the lateral collateral ligament posteriorly.

The third layer contains the lateral joint capsule which attaches on the femoral and tibial articular boundaries. Bursae and soft tissues lie between the iliotibial band and lateral menisci. As such, parameniscal cysts extending from the lateral meniscus may cause friction with the iliotibial band, resulting in the hallmark inflammation and pain of ITBS (Figure 2).

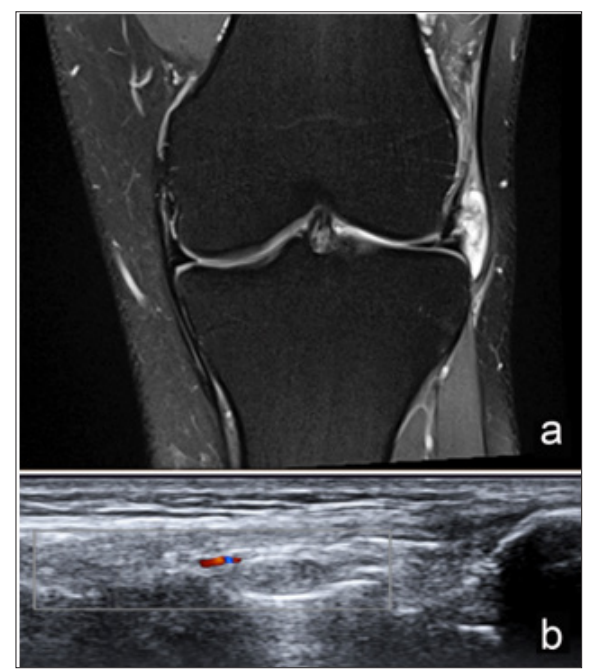

Figure 2: As such, parameniscal cysts extending from the lateral meniscus may cause friction with the iliotibial band, resulting in the hallmark inflammation and pain of ITBS.

\section{Diagnosis}

Meniscal cysts can be asymptomatic. However, symptomatic cysts usually present with symptoms typical of knee and/or meniscal injuries. Symptoms include pain, limited range of motion, swelling, tenderness along the joint line, and presence of a palpable mass along the joint line [1,2]. MRI is the modality of choice for detecting meniscal injuries [3]. In a study of 57 patients, researchers Crues et al. reported a sensitivity and specificity both of $92 \%$ for meniscal pathology on MRI as compared to arthroscopy [11]. On MRI, clinicians identify parameniscal cysts as fluid-intense lesions with clear connections to the adjacent meniscus [12]. Ultrasound is also useful for radiologic evaluation of meniscal cysts. In a study of 50 patients with a palpable mass, researchers Rutten et al reported a sensitivity and specificity of $97 \%$ and $86 \%$ respectively for meniscal cysts. (In the same study, researchers calculated a PPV of $94 \%$ and a NPV of $92 \%$.) Clinicians identify parameniscal cysts as hypoechoic cysts in contact with the meniscus on ultrasound [4].

Parameniscal cysts remain a rare finding. In a study of 276 patients with knee pain evaluated with ultrasound, Artur et al., reported parameniscal cysts in $<1 \%$ of patients [13]. ITBS is commonly seen in running, soccer, cycling and other sports involving repetitive extension and flexion of the knee. It is a diagnosis of exclusion. Ober's test may be used to confirm the diagnosis. The test involves pressing the lateral femoral condyle during movement at the knee joint while the hip is abducted resulting in a positive finding of sharp, burning pain [7].

\section{Treatment}

Treatment is based on the underlying pathology that led to cyst formation. Most commonly, meniscal tears require surgery to prevent recurrence of the meniscal cyst (Figure 3). The most common surgical technique is an arthroscopic partial meniscectomy with cyst decompression [1-2,14]. Reagan et al. concluded that $80 \%$ of their patients who underwent combined partial meniscectomy and open cyst excision, went on to report good-to-excellent results. Only $50 \%$ of patients who underwent partial meniscectomy alone reported good-to-excellent results [1]. In cases without associated meniscal tear, some clinicians utilize percutaneous decompression and debridement to optimize meniscal tissue preservation [1,2]. An alternative treatment option is ultrasound-guided percutaneous aspiration which is both simpler and less invasive. MacMahon et al. studied 18 patients who received ultrasound-guided percutaneous aspiration for lateral meniscal cysts [15]. Ten patients reported complete resolution of symptoms, two patients reported satisfactory sustained response with occasional pain, and the remaining six patients were pain-free between 1-8 weeks before pain returned.

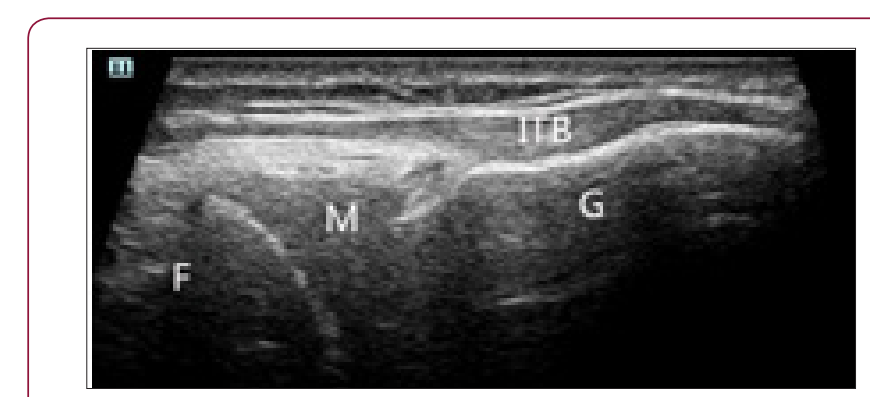

Figure 3: Most commonly, meniscal tears require surgery to prevent recurrence of the meniscal cyst.

Ultrasound-guided percutaneous aspiration is a well-tolerated and safe procedure with good clinical outcomes.

Initial treatment for ITBS attempts to limit the movements responsible for the irritation and to reduce inflammation. These strategies include reducing activity, applying ice or heat to the affected area, stretching, the use of NSAIDs and lastly, injection of corticosteroids $[7,16]$. In refractory cases, surgery may be considered. Multiple authors reported that conservative therapies have success rates as high as $94 \%[7]$. 


\section{Technique}

Our institution examined cases of patients who presented with both anterolateral parameniscal cysts together with Iliotibial Band Syndrome. We used ultrasound to confirm the diagnosis of parameniscal cysts and provide guidance for aspiration and injection of a steroid-anesthetic mixture. Operators performed scans with an 18-5 MHz linear transducer and an 11-3 MHz Resona 7 (Mindary Healthcare) linear transducer, on an Acuson S3000 scanner (Siemens Healthcare). Patients were positioned on their side or supine, with the hip in neutral flexion and the knee at $30^{\circ}$ to $45^{\circ}$ degrees of flexion. To begin, the transducer was placed over the anterior knee on the long axis to the patellar tendon. We then scanned laterally and first identified the iliotibial band in the long axis of the coronal plane. We followed the iliotibial band from above the lateral femoral condyle to its insertion on Gerdy's tubercle, located on the proximal lateral tibia (Figure 4). Of note, the iliotibial band is separated from the lateral margin of the knee by the lateral synovia recess and the ITB bursa. The lateral synovial recess is a layer of richly innervated and vascularized adipose tissue deep to the ITB.

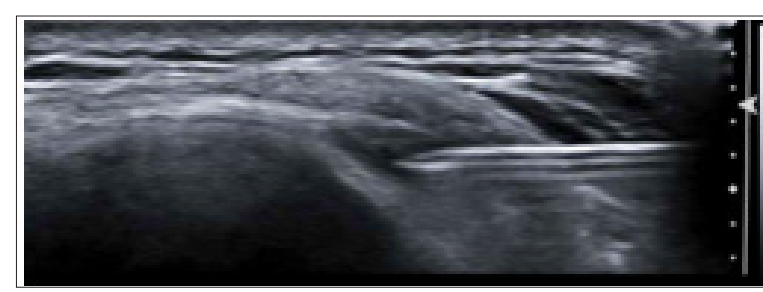

Figure 4: We followed the iliotibial band from above the lateral femoral condyle to its insertion on Gerdy's tubercle, located on the proximal lateral tibia.

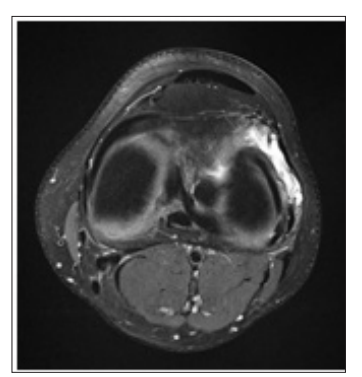

Figure 5: The same needle is then advanced under direct ultrasound guidance into the parameniscal cyst along the anterolateral margin of the lateral meniscus

The ITB bursa is an adventitial bursal extension from the synovial capsule. When identifying the ITB bursa between the ITB and lateral femoral condyle, it is important to evaluate the tissues between the iliotibial tract and the distal femur more proximally for disorders related to ITBS. The anterior horn of the lateral meniscus is deep in the iliotibial tract. Following identification of the paramenical cysts, researches injected an anesthetic corticosteroid mixture of $1 \mathrm{~mL}$ ( $40 \mathrm{mg} / \mathrm{mL}$ ) of triamcinolone (Kenalog; Apothecon, Bristol-Myers Squibb Co, Princeton, NJ), $0.5 \mathrm{~mL}$ of $1 \%$ lidocaine, and $0.5 \mathrm{~mL}$ of $0.5 \%$ bupivacaine (Sensorcaine; AstraZeneca Pharmaceuticals, Wilmington, DE). Anesthesia of patients' skin was achieved with injection of $1 \mathrm{~mL}$ of $1 \%$ lidocaine through a 25-gauge, 1.5-in hypodermic needle using a sterile technique. The same needle is then advanced under direct ultrasound guidance into the parameniscal cyst along the anterolateral margin of the lateral meniscus (Figure 5). Then, another 25-gauge needle was placed into the undersurface of the adjacent ITB. Once the both needles were in the correct position, the therapeutic mixture was injected. Distention of the bursae was the determining factor for a successful injection.

\section{Discussion}

Historically, symptomatic parameniscal cysts were treated with arthroscopic meniscectomy and either a percutaneous or arthroscopic cystectomy [17-19]. With regards to ITBS, experts differ as to when surgical treatment should be implemented. Martens et al. suggests that conservative treatments should be maintained for an average of 9 months before consideration of surgical intervention [20]. Others favor surgical intervention based on the physical finding of increased pain at 30 degrees flexion (ie., when the posterior fibers of the ITB are pulled more tightly against the lateral femoral condyle than the more anterior fibers). In this case, a surgical release in the posterior fibers may be needed to correct the problem [21,22]. However, surgical intervention of this nature necessitates general anesthesia. Conservative treatment remains first line therapy for ITBS. At present, musculoskeletal ultrasound (MSKU) has emerged as an important imaging diagnostic modality in areas of sports medicine [23].

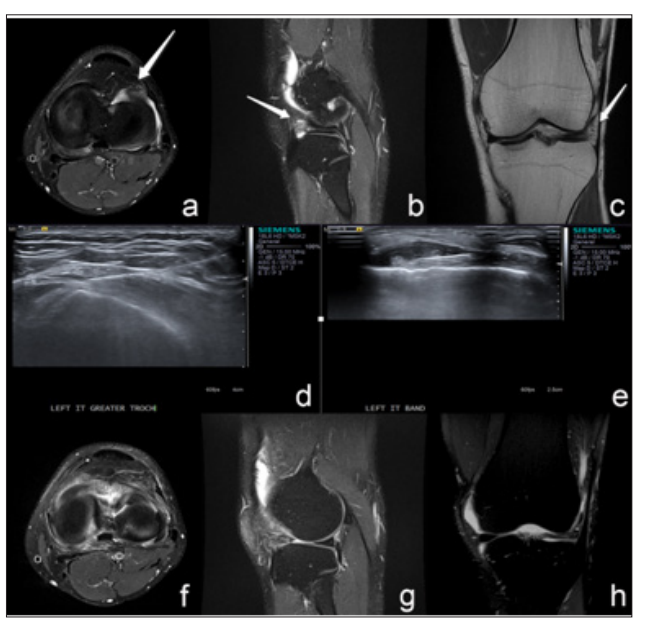

Figure 6: Arthroscopy has a very high sensitivity and specificity as the gold standards for diagnosing injury.

MSKU can be used to quantitatively observe knee movement. MSKU is also a non-invasive, low-cost procedure, making it easier to obtain compared with other tests [24]. Arthroscopy has a very high sensitivity and specificity as the gold standards for diagnosing injury (Figure 6). However, it is both costly and invasive [25,26]. MSKU with high frequency probes can be used to clearly visualize the soft tissues around the knee and the peripheral edge of the menisci. MSKU can be used to easily guide needle placement for parameniscal cyst aspiration and injection for treatment of ITBS. With the advantages of easy operation, high accuracy, flexible use 
of in-plane and out-plane needle movement, ultrasound-guided treatment offers a strong alternative to invasive arthroscopic surgery. Although ultrasound-guided parameniscal cyst aspiration in patients with associated ITBS drug injection is promising, limitations exist. The operator needs to be skilled. He or she must guarantee the accuracy of the needle can and take care to avoid the common peroneal nerve.

\section{Conclusion}

Anterolateral parameniscal cysts with associated ITBS is a cause of great morbidity. The mainstay of treatment is invasive surgical repair. We demonstrate a novel technique that has proven safe and well tolerated. There were no short or long-term complications following the procedure. By aspirating the cyst under ultrasound and injecting an anesthetic corticosteroid mixture to reduce the acute inflammatory response, subjects can achieve pain relief without the need for arthroscopy repair of the lateral meniscal tear. Ultrasound guidance can provide direct visualization of parameniscal cysts and confirm correct injection placement, resulting in increased accuracy and more successful patient outcomes. Ultrasound guided parameniscal cyst aspiration with corticosteroid injection is a far less invasive and equally effective treatment modality, which is a safer compared with arthroscopic surgery, and achieves similar long-term results.

\section{Acknowledgement}

This project was supported by the Clinical Research Project of Shenzhen People's Hospital(No. SYLY201702).

\section{References}

1. Anderson JJ, Connor GF, Helms CA (2010) New observations on meniscal cysts. Skeletal Radiol 39(12):1187-1191.

2. Bellisari G, Samora W, Klingele K (2011) Meniscus tears in children. Sports Med Arthrosc 19(1):50-55.

3. Nguyen JC, Arthur A De Smet, Ben K Graf, Humberto G Rosas (2014) MR imaging-based diagnosis and classification of meniscal tears. Radiographics 34(4): 981-999.

4. Rutten MJ, Collins JM, Van Kampen A, Jager GJ (1998) Meniscal cysts: detection with high-resolution sonography. AJR Am J Roentgenol 171(2): 491-496.

5. Bergin D, Hochberg H, Zoga AC, Qazi N, Parker L, et al. (2008) Indirect soft-tissue and osseous signs on knee MRI of surgically proven meniscal tears. AJR Am J Roentgenol 191(1): 86-92.

6. Fairclough J, Hayashi K, Toumi H, Lyons K, Bydder G, et al. (2006) The functional anatomy of the iliotibial band during flexion and extension of the knee: implications for understanding iliotibial band syndrome. J Anat 208(3):309-316.

7. Fairclough J, Hayashi K, Toumi H, Lyons K, Bydder G, et al. (2007) Is iliotibial band syndrome really a friction syndrome? J Sci Med Sport 10(2):74-76.
8. Beals C, Flanigan D (2013) A review of treatments for iliotibial band syndrome in the athletic population. Journal of Sports Medicine 367169.

9. Richard Ellis, Wayne Hing, Duncan Reid (2007) Iliotibial band friction syndrome-A systematic review. Manual Therapy 12: 200-208.

10. Irizarry JM, Recht MP (1997) MR imaging of the knee ligaments and the postoperative knee. Radiologic Clinics of North America 35(1): 45-76.

11. Crues JV, Ryu R, Morgan FW (1990) Meniscal pathology. The expanding role of magnetic resonance imaging. Clin Orthop Relat Res (252): 80-87.

12. Crowell MS, Westrick RB, Fogarty BT (2013) Cysts of the lateral meniscus. Int J Sports Phys Ther 8(3): 340-348.

13. Artul S, Khazin F, Hakim J, Habib G (2014) Ultrasonographic findings in a large series of patients with knee pain. Journal of Clinical Imaging Science 4(1): 45 .

14. Lu KH (2006) Arthroscopic meniscal repair and needle aspiration for meniscal tear with meniscal cyst. Arthroscopy 22(12):1367.e1-4.

15. Mac Mahon PJ, Brennan DD, Duke D, Forde S, Eustace SJ (2007) Ultrasound-guided percutaneous drainage of meniscal cysts: preliminary clinical experience. Clinical Radiology 62: 683-687.

16. Sangkaew C (2007) Surgical treatment of iliotibial band friction syndrome with the mesh technique. Arch Orthop Trauma Surg 127(4): 303-306.

17. Lantz B, Singer KM (1990) Meniscal cysts. Clin Sports Med 9(3): $707-$ 725 .

18. Ryu RK, Ting AJ (1993) Arthroscopic treatment of meniscal cysts. Arthroscopy 9(5): 591-595.

19. Glasgow MM, Allen PW, Blakeway C (1993) Arthroscopic treatment of cysts of the lateral meniscus. J Bone Joint Surg Br 75(2): 299-302.

20. Martens M, Libbrecht P, Burssens A (1989) Surgical treatment of the iliotibial band friction syndrome. The American journal of sports medicine 17(5): 651-654.

21. Noble CA (1980) Iliotibial band friction syndrome in runners. The American journal of sports medicine 8(4): 232-234.

22. Noble CA (1979) The treatment of iliotibial band friction syndrome. British journal of sports medicine 13(2): 51-54.

23. Henderson RE, Walker BF, Young KJ (2015) The accuracy of diagnostic ultrasound imaging for musculoskeletal soft tissue pathology of the extremities: a comprehensive review of the literature. Chiropractic \& manual therapies 23(1): 31

24. Grzelak P, Podgórski MT, Stefańczyk L, Domżalski M (2015) Ultrasonographic test for complete anterior cruciate ligament injury. Indian journal of orthopaedics 49(2): 143.

25. Kam CK, Chee DW, Peh WC (2010) Magnetic resonance imaging of cruciate ligament injuries of the knee. Canadian Association of Radiologists Journal 61(2): 80-89.

26. Seon JK, Park HW, Yoo SH, Song EK (2016) Assessing the accuracy of patient-specific guides for total knee arthroplasty. Knee Surgery, Sports Traumatology, Arthroscopy 24(11): 3678-3683. 
ISSN: 2574-1241

DOI: $10.26717 / B J S T R .2018 .09 .001830$

Jean Jose Do. Biomed J Sci \& Tech Res

(C) This work is licensed under Creative

Submission Link: https://biomedres.us/submit-manuscript.php

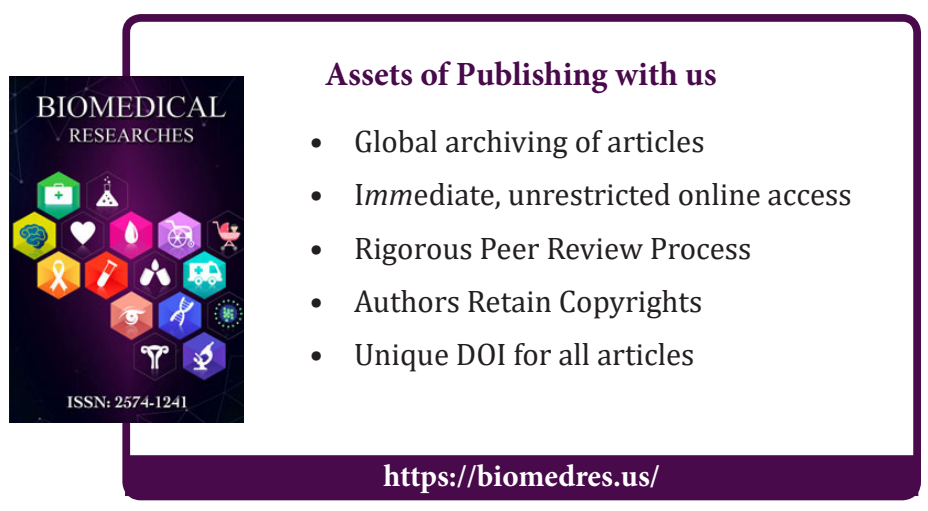

